Meta

Journal des traducteurs

Translators' Journal

\title{
L'enseignement théorique dans un programme de formation universitaire en traduction
}

\section{Marie-Christine Aubin}

Volume 48, numéro 3, septembre 2003

Traduction et enseignement

Translation and teaching

URI : https://id.erudit.org/iderudit/007604ar

DOI : https://doi.org/10.7202/007604ar

Aller au sommaire du numéro

Éditeur(s)

Les Presses de l'Université de Montréal

ISSN

0026-0452 (imprimé)

1492-1421 (numérique)

Découvrir la revue

Citer cet article

Aubin, M.-C. (2003). L’enseignement théorique dans un programme de formation universitaire en traduction. Meta, 48(3), 438-445.

https://doi.org/10.7202/007604ar
Résumé de l'article

Si on prend le terme théorie dans son sens de " construction intellectuelle méthodique et organisée », il ne fait aucun doute que celle-ci doit permettre aux traducteurs, à une époque où tout s'accélère, de travailler plus vite et plus efficacement. En effet, la théorie soutient la pratique et n'apparaît le plus souvent que dans ses résultats dans les programmes de formation, permettant aux étudiants d'accélérer leur acquisition des compétences essentielles à la pratique. Autrement dit, si la théorie n'existait pas, il faudrait l'inventer car aucun programme de formation ne serait viable sans une réflexion théorique sur la pratique du métier. 


\title{
L'enseignement théorique dans un programme de formation universitaire en traduction
}

\author{
MARIE-CHRISTINE AUBIN \\ Collège universitaire de Saint-Boniface, Saint-Boniface, Canada \\ amcaubin@aol.com
}

\begin{abstract}
RÉSUMÉ
Si on prend le terme théorie dans son sens de «construction intellectuelle méthodique et organisée », il ne fait aucun doute que celle-ci doit permettre aux traducteurs, à une époque où tout s'accélère, de travailler plus vite et plus efficacement. En effet, la théorie soutient la pratique et n'apparaît le plus souvent que dans ses résultats dans les programmes de formation, permettant aux étudiants d'accélérer leur acquisition des compétences essentielles à la pratique. Autrement dit, si la théorie n'existait pas, il faudrait l'inventer car aucun programme de formation ne serait viable sans une réflexion théorique sur la pratique du métier.
\end{abstract}

\section{ABSTRACT}

Theory can be considered as an organized set of principles devised to explain facts or phenomena. Translatology - the theory of translation - therefore should help professional translators perform faster and more efficiently, especially at a time when everything seems geared to speed. Theory and practice go hand in hand. In training programs, the methodology used to impart translation skills draws heavily on theory. In other words, if theory did not exist, it would have to be invented, since no program could survive without some theorizing on the practice of the profession.

\section{MOTS-CLÉS/KEYWORDS}

théorie, pratique, programmes, formation, enseignement de la traduction

Malgré tous les efforts que la plupart des universités et leur programme de traduction ont multipliés pour ancrer leur enseignement dans la pratique, on entend toujours répéter ce même cliché: "On apprend la théorie à l'université et ce n'est qu'après avoir quitté l'université qu'on apprend la pratique du métier.» Si l'on en croit ce cliché, il y aurait deux mondes, le monde de la théorie, celui de l'université, et le monde de la pratique, celui des professionnels de la traduction qui œuvrent dans des bureaux spécialisés. Présentés ainsi, ces deux mondes apparaissent distincts, séparés l'un de l'autre par un fossé infranchissable. Pire, la formation se trouve dévalorisée, accusée d'être inadaptée à la réalité du métier.

Mais qu'en est-il exactement? Qu'entend-on par théorie et par pratique? La théorie est-elle effectivement l'apanage des universitaires? Les traducteurs en exercice n'ont-ils donc aucune activité théorique? Enfin, quelle est la part de la théorie et de la pratique dans nos programmes de formation?

Il faut d'abord s'entendre sur une définition de ce qu'est la théorie dans le contexte de la traduction. Le Robert fait état de deux acceptions modernes du terme «théorie». C'est, d'une part, «un ensemble d'idées, de concepts abstraits, plus ou moins organisés, appliqués à un domaine particulier» (dans ce sens, le mot renvoie à 
«spéculation», «doctrine», «conception»); d'autre part, c'est une «construction intellectuelle méthodique et organisée, de caractère hypothétique (du moins dans certaines de ses parties) et synthétique» et le terme renvoie alors à «hypothèse» ou «système».

Quant à "pratique», Le Robert lui donne, parmi ses nombreuses acceptions, les définitions suivantes susceptibles de s'appliquer à la traduction: «Activités volontaires visant des résultats concrets (opposé à théorie)». Dans ce sens, la pratique s'associe à «empirique», "expérimental», "pragmatique». Deuxièmement, la pratique, c'est aussi une «manière concrète d'exercer une activité (opposé à règle, principe), par exemple: la pratique d'un art.» Ce sens renvoie à «expérience» et donne, dans sa forme adjectivale, l'association «exercices pratiques» que l'on emploie beaucoup en didactique et en pédagogie.

La théorie que l'on retrouve dans les programmes de formation en traduction correspond plutôt au deuxième sens indiqué plus haut - particulièrement au premier cycle -, et c'est dans cette perspective que nous l'abordons ici. Ne devons-nous pas en effet tout faire pour que nos étudiants parviennent à construire leur savoir et leur savoir-faire de façon méthodique et organisée? En quoi consistent donc ces théories de la traduction et qu'apportent-elles à l'enseignement de cette discipline?

En traduction, les théories touchent aux questions suivantes: la structure et le fonctionnement des langues, la comparaison des langues, le processus cognitif de l'activité traduisante.

La structure et le fonctionnement des langues. Cette réflexion relève de la linguistique, ou, plus précisément de la lexicologie, de la terminologie et de la syntaxe. On s'interroge sur les rapports entre linguistique et traduction, sur le langage et la connaissance des langues, sur les systèmes linguistiques et la capacité de traduire. Cette réflexion sous-tend l'affirmation de Jost Tier, selon laquelle «chaque langue crée une image de la réalité, complète, et qui se suffit à elle-même. Chaque langue structure la réalité à sa façon et, par là même, établit les éléments de la réalité qui sont particuliers à cette langue donnée» (cité par Mounin 1963: 44). C'est ainsi qu'on retrouve une pléthore de termes pour nommer et caractériser la neige dans les pays nordiques, mais non dans les pays où le climat est plus doux.

La comparaison des langues. Issue d'études linguistiques ou stylistiques, cette comparaison des systèmes linguistiques et socio-linguistiques a donné le comparatisme, celui de Vinay et Darbelnet (1958), dont l'ouvrage a été par la suite très controversé mais dont les concepts sont restés un pilier de notre réflexion. Les langues sont considérées comme l'expression de visions du monde propres aux pays qui les parlent et l'accent est mis sur les emplois sociaux du langage: niveaux de langue, interférences en cas de contacts fréquents (anglicismes). Ce comparatisme a évolué vers des études contrastives, mieux ancrées dans l'analyse contextuelle. Toutes aboutissent à la constatation qu'une traduction doit en fait toujours être une adaptation dont le fondement n'est pas linguistique mais culturel. C'est ce qu'exprime Dominique Aury, en préface au livre de Mounin: «L'ensemble de l'expérience pour un peuple ou pour un pays donné, que les ethnologues appellent culture, ne recouvre jamais entièrement un autre ensemble, fût-ce dans l'ordre purement matériel: on ne traduit pas dollar, on ne traduit pas rouble, parce que la chose en France et en français n'existe pas» (1963: X). Au XXI ${ }^{\text {e }}$ siècle, cette conception sur les variances culturelles indispensables à l'activité traductionnelle aboutit même à scinder les concepts de traduction et de 
«localisation », cette dernière se voulant plus ciblée sur le public qui devra la recevoir. Cette distinction relancera le débat sur la question de la fidélité mais dans une perspective renouvelée, beaucoup plus pragmatique que celle de la traduction des textes littéraires. À l'extrême, la notion de l'impossibilité de traduire a pu être avancée car, Malraux l'affirme, "en profondeur, chaque civilisation est impénétrable pour les autres $^{1} »$. À l'autre extrêmité, la localisation permet des adaptations fort éloignées du texte initial.

Le processus cognitif de l'activité traduisante. Ces recherches ont pu susciter des débats entre sourciers et ciblistes, mais elles partent le plus souvent de l'observation empirique du traducteur ou de l'interprète en train de traduire ou d'interpréter. C'est ainsi que naît la théorie du sens sous la plume de Seleskovitch ou sous celle de Lederer:

Les unités de sens se succèdent en se chevauchant dans l'esprit de l'interprète de simultanée pour produire le sens général, elles se transforment en connaissances déverbalisées au fur et à mesure qu'elles s'intègrent en des unités plus vastes, en des idées plus conséquentes. [...] L'unité de sens n'existe qu'au plan du discours; elle ne se confond pas avec des mots, des syntagmes, des collocations ou des figements. Représentation mentale, elle correspond au plan psychique à un état de conscience de courte durée (Lederer 1994: 27-28).

De même, le traducteur passe par une étape de déverbalisation, où les unités de sens existent dans son esprit mais sans être attachées à une formulation, ni dans la langue de départ ni dans la langue d'arrivée. Ce n'est qu'ensuite que la réexpression du sens se fait en langue d'arrivée, avec la même spontanéité raisonnée de l'écrivain cherchant ses mots pour bien exprimer sa pensée.

Toutes les activités mentionnées ci-dessus ont entraîné la création d'un métalangage, terminologie particulière propre au domaine, servant à décrire les diverses activités du processus de traduction, mais aussi à qualifier les traductions elles-mêmes ( "procédés» et erreurs) et à les évaluer. L'évaluation de la qualité des traductions est en soi un champ entier de recherches, lequel, on s'en doute, ne peut qu'avoir des répercussions sur l'enseignement et sur la pratique de la traduction. On se pose entre autres la question de la fidélité par rapport au texte de départ, de la liberté du traducteur, question résumée ainsi par Marianne Lederer:

L'exigence de littéralisme de certains traductologues rappelle l'interrogation vieille de plusieurs siècles: le traducteur doit-il être libre ou fidèle? L'alternative ainsi posée est fausse car chacun de ses termes, "fidélité", "liberté", ambitionne de s'appliquer à l'ensemble d'un texte, alors que toute traduction comporte une alternance entre des correspondances (fidélité à la lettre) et des équivalences (liberté à l'égard de la lettre) (1994: 83).

Le métalangage, fruit des recherches théoriques, a entrainé à son tour d'autres activités de recherche: d'abord, l'organisation méthodique de la matière, notamment à des fins pédagogiques; c'est ce que Jean Delisle a entrepris dans son manuel $\mathrm{La}$ traduction raisonnée (1993). Ensuite, l'analyse du discours et l'analyse comparée des discours du texte de départ et du texte d'arrivée sans laquelle aucune évaluation de la qualité ne serait possible (voir Seleskovitch, Lederer, Delisle). Enfin, l'élaboration de méthodes d'évaluation des traductions, champ de recherche découlant de tout ce qui précède. Notons, dans cette catégorie, les travaux de Daniel Gouadec qui ont abouti 
à l'établissement du SICAL, système d'évaluation des traductions adopté par le Bureau de la traduction du gouvernement fédéral canadien et par d'autres organismes d'évaluation: Conseil des traducteurs et interprètes du Canada (CTIC), services de traduction de diverses provinces. Ces recherches ont eu une application pratique tangible dans le quotidien des traducteurs en exercice. De nos jours, elles s'intègrent encore dans ce que l'on appelle les «contrôles de qualité».

Par ailleurs, il faut ajouter à ces recherches, toutes utiles, selon moi, pour comprendre les diverses facettes du travail de traduction, les études, plus sociales, sur l'intégration de la traduction dans la société en général ou dans des sociétés particulières; l'étude des marchés qui s'ouvrent à la traduction ou à la localisation; l'importance de la traduction pour une discipline en particulier (la littérature, les sciences, le commerce international, etc.); l'étude du rôle et des responsabilités du traducteur vis-à-vis de son client et du public, recherches qui ont abouti à la mise en place d'un code de déontologie pour la profession.

Enfin, l'informatique a induit une toute nouvelle avenue de recherches, lesquelles viennent parfois infirmer ou confirmer des études théoriques antérieures partant d'hypothèses sur la langue ou sur des principes d'équivalence. C'est ainsi que, selon les hypothèses favorisées par des concepteurs d'outils de traduction automatique, on a pu assister à l'échec total de ces outils ou à leur relatif succès. Ainsi, les outils se fondant sur le mot comme base d'équivalence ont donné, dans la plupart des cas, des résultats désastreux, attribuables entre autres, à l'impossibilité de ces systèmes d'accorder les mots variables d'une phrase. Ceux fondés sur les équivalences, largement employés dans les machines multilingues comme les distributeurs de billets, donnent de meilleurs résultats, quoiqu'ils soient très limités dans leurs applications et qu'ils ne puissent générer de traductions nouvelles, créatives. Ces recherches se sont concrétisées par l'élaboration d'outils de traduction automatique ou d'aide à la traduction.

Les premiers logiciels de traduction automatique, créés à partir de l'hypothèse que la traduction était une recherche de correspondance de mots, se sont vite révélés inadéquats, puisque ni le sens général du texte ni les caractéristiques syntaxiques indispensables ne pouvaient se transférer correctement dans le texte d'arrivée, confirmant ce faisant que l'activité de traduction était une recherche et une réexpression du sens et non des mots. Même les erreurs sont utiles lorsqu'elles permettent de faire avancer les choses.

Plus intéressantes dans leurs résultats, les recherches statistiques sur les fréquences de traductions d'un mot en particulier et sur l'utilisation de trinômes comme base de création de logiciels de traduction automatique, ont donné naissance à des prototypes de traduction automatique très convaincants, quoique difficilement utilisables à grande échelle.

Comme nous le voyons, les recherches théoriques aboutissent le plus souvent à des réalisations pratiques: dictionnaires et lexiques, qu'ils soient sous forme de livres ou de logiciels; bases de données terminologiques multilingues; concordanciers. Que l'on parle de TERMIUM, du Grand dictionnaire terminologique (granddictionnaire. com), de TransSearch (pour ne parler que des outils canadiens), ou que l'on considère les grammaires et ouvrages spécialisés de toutes sortes, ces outils sont utilisés au quotidien par les traducteurs en exercice et ne sont donc pas de pures spéculations dont la profession pourrait se passer. De même, les recherches sur les méthodes d'évaluation ont permis de mettre en place des systèmes pratiques et efficaces de 
contrôle de la qualité qui donnent aux praticiens des outils fiables pour l'embauche et l'encadrement des jeunes traducteurs, outils plus rationnels que le recours à des méthodes empiriques. Enfin, la traductologie a su organiser la matière de façon à en faciliter l'apprentissage et l'enseignement.

Compte tenu de ce constat, nous pouvons donc nous demander pourquoi certains praticiens ne perçoivent pas cet apport de la recherche théorique dans leur quotidien. Comment s'organise donc le quotidien d'un traducteur en exercice?

De nos jours, l'informatique, même si elle est parfois perçue comme pesante par certains traducteurs, est présente dans la plupart des activités reliées à la traduction: réception électronique du texte dans un format défini (le plus souvent Word ou WordPerfect), maintien du format pour le texte traduit, d'où l'utilisation du même traitement de texte pour faire sa traduction, consultation d'outils (dictionnaires, anciennes traductions, banques terminologiques, concordanciers) en intranet ou par Internet, recherches lexicales ou documentaires. Si la consultation des livres reste importante, elle tient maintenant une place réduite dans la journée du traducteur. Quoi qu'il en soit, qu'il consulte des livres ou des outils informatisés, le traducteur doit bien, dans l'exercice de son métier, utiliser les produits de la recherche universitaire et faire lui-même de la recherche, recherche qu'il consigne souvent dans des fiches, terminologiques ou traductionnelles, qui viendront peut-être un jour enrichir une base de données ou créer de nouveaux outils: lexique d'un domaine en particulier, banque terminologique interne ou commercialisée, nouvelle édition du Guide du rédacteur ou du Français au bureau.

Finalement, pour résumer, on peut dire qu'à une époque où tout s'accélère, la théorie permet aux traducteurs de travailler plus vite et plus efficacement. Loin d'être scindées, les activités théoriques et les activités pratiques reliées à la traduction apparaissent comme des activités complémentaires dans l'exercice de la profession. Tous les traducteurs utilisent le fruit du travail des chercheurs et apportent aussi leur contribution à la recherche, notamment en terminologie. Leurs systèmes d'évaluation sont établis à partir du repérage et du classement des erreurs décrites par les chercheurs. Malgré ce constat, la perception exprimée par le cliché nous pousse à nous demander si la part faite à la théorie dans les programmes de formation ne serait pas trop grande, disproportionnée par rapport à sa place dans l'exercice du métier.

Il faut tout d'abord distinguer la place qu'occupe la réflexion théorique chez les professeurs de la théorie en tant que matière d'enseignement au sein des programmes. Si la réflexion théorique est si prisée dans les universités, c'est bien parce qu'elle aboutit soit à des réalisations qui peuvent avoir des répercussions positives dans la société, soit qu'elle oriente l'enseignement de la discipline. Pour ce qui est de la traduction, la réflexion théorique sur l'activité traduisante sous-tend l'élaboration d'une méthode de travail et d'une méthode d'enseignement qui permettront aux étudiants d'acquérir, par étapes ou par objectifs, les différentes compétences indispensables pour mener à bien cette méthode de travail. La réflexion théorique des enseignants se trouve donc à la base d'une méthode d'enseignement organisée et efficace qui évite aux étudiants les tâtonnements et les détours par lesquels eux-mêmes sont peut-être passés.

Une organisation cohérente de la matière commence par l'apprentissage du langage qui donnera aux étudiants les moyens linguistiques de s'exprimer clairement sur les diverses activités reliées à la discipline. Au fil du temps, le métalangage de la 
traduction s'est élaboré, par le travail des philologues, linguistes, grammairiens et autres spécialistes de langues, pour décrire le fonctionnement de celles-ci et les manières de les traduire, bonnes et mauvaises. Ainsi, le classement des activités reliées à la traduction et l'apprentissage du langage qui y correspond rendent l'apprentissage des contenus théoriques de base plus rapides et plus efficaces. De plus, cette étude méthodique et organisée s'applique aussi aux domaines connexes à la traduction comme la lexicologie, la terminologie, la linguistique, l'analyse du discours, la grammaire et la syntaxe, en facilitant la compréhension et l'apprentissage.

Ces recherches, en plus de classer systématiquement l'activité traduisante en objectifs pédagogiques progressifs, permettent aussi, nous l'avons vu, de créer des outils, non seulement d'aide à la traduction, mais aussi des didacticiels, manuels et livres d'exercices qui aideront les étudiants à acquérir concepts et pratique dans le cadre de leurs études. La partie recherche de la charge des professeurs a donc bien sa raison d'être et sa pertinence et ce, tout autant quand il s'agit d'enseigner une discipline essentiellement pratique dans sa réalisation comme c'est le cas de la traduction que pour l'enseignement de sciences pures. Mais qu'en est-il de l'étudiant lui-même? Doit-il lui aussi aborder toutes ces questions?

Certaines universités proposent à leurs étudiants des cours spécifiquement intitulés "Théories de la traduction", mais c'est plutôt au deuxième cycle que ces cours sont offerts. La part de la théorie enseignée au niveau du premier cycle est très limitée et intégrée aux divers cours qui constituent les programmes. On y enseigne ainsi le métalangage de la traduction, d'abord globalement, puis au fil de l'apprentissage des diverses activités reliées à la traduction (terminologie, lexicologie, grammaire, rédaction) et des cours de traduction comme telle (notamment par le réemploi de la terminologie exacte pour qualifier les erreurs ou les procédés utilisés) ${ }^{2}$.

On présente aussi des notions et des réflexions sur l'activité traduisante et sur le rôle et les responsabilités du traducteur vis-à-vis de son client, de la langue, de l'auteur du texte qu'il traduit, etc. On présente encore les notions de base de linguistique, de lexicologie, de terminologie et d'analyse du discours, notions sans lesquelles la pratique ne pourrait s'acquérir (il faut en effet comprendre la notion de champ sémantique pour bien remplir une fiche terminologique). Enfin, et cela tient encore au métalangage, le perfectionnement en grammaire passe aussi par les notions théoriques qui servent à décrire le fonctionnement de la langue.

On peut donc affirmer que la part de la théorie dans nos programmes est non seulement très réduite mais limitée aux concepts les plus indispensables à l'exercice du métier. Tâchons maintenant d'évaluer le contenu pratique de nos programmes.

Il faut distinguer ici les cours de traduction et les cours de disciplines connexes. Dans les cours de disciplines connexes (grammaire, rédaction, perfectionnement linguistique, lexicologie ou syntaxe comparée, terminologie), la part de traduction comme telle est très variable: elle peut être inexistante dans des cours de grammaire ou de rédaction, soit française, soit anglaise, ou tenir une certaine place, comme dans les cours de lexicologie ou de syntaxe comparée. Nous n'en tiendrons pas compte pour le calcul qui suit.

Dans les cours de traduction véritable, la pratique se fait de diverses manières: par des exercices portant sur des objectifs particuliers, par la traduction de textes dans le cadre de séminaires, par la traduction de textes en vue d'une évaluation sommative, traduction réalisée soit à la maison (devoirs) soit en classe s'il s'agit de 
tests sur table pour lesquels les textes seront à traduire en temps limité. Il est certain que le nombre de mots traduits dans le cadre d'un cours de traduction peut varier considérablement en fonction du nombre d'étudiants inscrits au cours et du niveau où se situe le cours dans l'apprentissage de la matière. Ainsi, il parait normal que les étudiants ne traduisent que 250 mots par semaine dans un cours de 3 crédits d'introduction à la traduction mais qu'ils traduisent le double de cela dans un cours de $4^{\text {e }}$ année. Si l'étudiant suit, bon an mal an, 12 crédits de cours de traduction sur les 30 suivis en tout (ces 30 crédits comptant 6 à 12 crédits de matières connexes et 6 à 12 crédits de matières d'option), il pourrait traduire 14000 mots dans sa première année de traduction et 28000 en dernière année. À cette expérience pratique (environ 63000 mots pour les trois années) viendra s'ajouter en fin de programme des stages au cours desquels il pourrait traduire 10 à 15000 mots (soit une moyenne de 500 mots par jour pendant six semaines), ce qui lui donnerait une expérience totale non négligeable d'environ 78000 mots pour un programme de baccalauréat spécialisé. Nous ne considérons même pas ici les programmes de travail-études (coop, programme symbiotique, etc.) pour lesquels les statistiques manquent, quoique l'expérience acquise par les étudiants dans ce type d'environnement ne puisse qu'augmenter les chiffres que nous donnons. Avec ou sans programme coop, le nombre total de mots traduits dans le courant des études ne peut évidemment pas se comparer à la production d'un traducteur en exercice, même débutant; malgré tout, elle représente, en nombre d'heures, une proportion importante du travail de l'étudiant.

En terminologie et en documentation, le travail de l'étudiant consiste entre autres à faire des recherches documentaires, à apprendre à lire les fiches que l'on trouve dans les diverses banques terminologiques et à réaliser eux-mêmes de telles fiches. Tous ces travaux sont bien de nature essentiellement pratique.

La formation universitaire est donc elle aussi axée sur la pratique et le travail théorique des professeurs ne vise qu'à renforcer la pertinence et l'efficacité des programmes et à augmenter la place disponible dans ces programmes pour la pratique. La théorie soutient donc la pratique et n'apparaît le plus souvent que dans ses résultats dans les programmes de formation, permettant aux étudiants d'acquérir les compétences essentielles à la pratique beaucoup plus rapidement que s'ils n'étaient soumis qu'aux tâtonnements de l'expérimentation pratique.

À la lumière de cette étude, il apparaît donc que, dans les universités comme dans l'exercice du métier de traducteur, théorie et pratique sont solidaires et complémentaires. Non seulement il n'y a pas de fossé entre les deux, ni entre les universités et les milieux professionnels, mais il y a une vraie symbiose qui permet, à chaque étape de la formation, de tirer le meilleur parti de cette formation au moyen d'une construction intellectuelle méthodique et organisée associée à une réflexion sur les méthodes employées.

On peut donc affirmer que si la théorie n'existait pas, il faudrait l'inventer, car aucun programme de formation ne serait viable sans une réflexion théorique sur la pratique du métier, celle des professeurs qui ont systématisé les notions à enseigner et celle des étudiants qui, même si elle est plus restreinte, accélère leur apprentissage et leur donne une idée des débats éthiques et sociaux associés au métier de traducteur. 


\section{NOTES}

1. La voie royale, cité par Mounin (1963).

2. Voir le détail des programmes canadiens sur le site de l'ACET (Association canadienne des écoles de traduction) : <http://www.uottawa.ca/associations/acet>.

\section{RÉFÉRENCES}

Delisle, J. (1984): L'analyse du discours comme méthode de traduction, Ottawa, Presses de l'Université d'Ottawa.

Delisle, J (1993): La traduction raisonnée, Ottawa, Presses de l'Université d'Ottawa.

GOUADEC, D. (1989): Le traducteur, la traduction et l'entreprise, AFNOR.

Larose, R. (1989): Théories contemporaines de la traduction, Sillery, Presses de l'Université du Québec.

Lederer, M. (1994): La traduction aujourd'hui. Paris, Hachette.

Mounin, G. (1963): Les problèmes théoriques de la traduction, Paris, Gallimard.

Vinay, J.-P. et J. Darbelnet (1958) : Stylistique comparée du français et de l'anglais, Paris, Didier. 